\title{
Women manifest more severe COPD symptoms across the life course
}

This article was published in the following Dove Press journal:

International Journal of COPD

\author{
Dawn L DeMeo' \\ Sreeram Ramagopalan ${ }^{2}$ \\ Abhishek Kavati ${ }^{3}$ \\ Ashok Vegesna ${ }^{3}$ \\ Meilan $\mathrm{K} \mathrm{Han}^{4}$ \\ Anthony Yadao ${ }^{5}$ \\ Teresa K Wilcox ${ }^{2}$ \\ Barry J Make ${ }^{6}$

\section{On behalf of COPDGene Investigators} \\ 'Channing Division of Network \\ Medicine and the Pulmonary and \\ Critical Care Division, Brigham and \\ Women's Hospital, Harvard Medical \\ School, Boston, MA, USA; ${ }^{2}$ Real-World \\ Evidence, Evidera, London, UK; ${ }^{3}$ US \\ Health Economics and Outcomes \\ Research, Novartis Pharmaceuticals \\ Corporation, East Hanover, NJ, \\ USA; ${ }^{4}$ Division of Pulmonary \\ and Critical Care, University of \\ Michigan, Ann Arbor, MI, USA; ${ }^{5}$ US \\ Clinical Development and Medical \\ Affairs, Novartis Pharmaceuticals \\ Corporation, East Hanover, NJ, USA; \\ ${ }^{6}$ Division of Pulmonary, Critical Care, \\ and Sleep Medicine, National Jewish \\ Health, Denver, CO, USA
}

Correspondence: Dawn L DeMeo Channing Division of Network Medicine and the Pulmonary and Critical Care Division, Brigham and Women's Hospital, Harvard Medical School, I8I Longwood Ave, Boston, MA 02115, USA Email redld@channing.harvard.edu
Background: Previous studies suggest that gender differences exist in COPD diagnosis and symptoms; these differences may be more pronounced in younger adults. Our objective was to explore age-associated gender differences across a range of COPD severities.

Materials and methods: A total of 4,484 current and former smokers with COPD from the Genetic Epidemiology of COPD cohort were investigated using regression modeling to explore the association between gender, age, disease severity, and the contributing elements of the Global Initiative for Chronic Obstructive Lung Disease (GOLD) classification system (symptoms, exacerbation risk, airflow limitation).

Results: The age-gender interaction was observed across multiple age categories. Compared to men with COPD, younger women with COPD had a greater likelihood of more severe dyspnea, airflow limitation, greater risk for exacerbations, and categorization in GOLD groups B and D. These differences were less pronounced in older women with COPD. However, older women remained more likely to experience severe dyspnea and to manifest more severe COPD (B vs A) than older men, despite lower pack-years of smoking.

Conclusion: These data demonstrate the significant symptom burden of COPD in women, especially younger women. More research is needed to understand the pathogenesis of increased severity of COPD in women and to develop gender-targeted clinical assessment and management approaches to improve outcomes for women and men with COPD at all ages.

Keywords: COPD, gender, age, symptoms, exacerbations

\section{Introduction}

COPD is a heterogeneous condition characterized by consistent and progressive airflow limitations. COPD is one of the most common chronic conditions in the USA, estimated to affect $\sim 5.7 \%$ of all adults, and in 2013 was identified as the third leading cause of death.

COPD has historically been viewed as a disease of men; however, prevalence and mortality have been increasing in women in recent decades. ${ }^{2}$ This has been attributed to increasing rates of smoking among women, ${ }^{3}$ although evidence suggests women may be more susceptible to the harmful effects of cigarette smoke and other known COPD risk factors. ${ }^{4}$ Indeed, gender differences have previously been observed in the clinical manifestations of COPD, with women reporting more severe dyspnea and a higher rate of exacerbations compared to men. ${ }^{5}$ Women and younger patients with COPD tend to report higher levels of physical and psychological symptoms. ${ }^{6}$ Women also are over-represented amongst subjects with severe, early-onset COPD. ${ }^{7,8}$ In a study of 2,500 current and former smokers in Genetic Epidemiology of COPD (COPDGene) cohort, female sex increased the risk of severe early-onset COPD by threefold. ${ }^{8}$ 
The predominance of women with early-onset COPD suggests that younger women may, in general, be more susceptible to severe manifestations of the disease, although more research is needed to clearly define age-by-gender interactions.

The COPD Global Initiative for Chronic Obstructive Lung Disease (GOLD) groups are a multidimensional approach to guide assessment and management of COPD by characterizing patients according to their symptoms, risk of exacerbation, and severity of airflow limitations. ${ }^{9}$ Although the GOLD classification scheme has been revised for 2017 , this symptom-based grading approach does have utility for considering associated features for the most symptomatic individuals. Research has begun to explore gender and age differences in the clinical features of COPD; however, no studies have taken a detailed approach to examine gender differences by age across GOLD grades and symptom groups. This information could help develop more effective strategies for the management of COPD symptoms across the life course; identifying gender-specific differences has been highlighted as a priority by the research community. ${ }^{10}$

The objective of the present study was to explore genderby-age interactions and disease severity as defined by GOLD groupings and the three key contributing elements of the GOLD classification system (symptoms, exacerbation risk, and airflow limitation severity) using data from a large wellphenotyped COPD cohort. A detailed approach was adopted to consider age by 5-year increments and to identify clinically relevant age cutoffs from which to examine these differences.

\section{Materials and methods Study design and population}

Participants were from the COPDGene study, which has been described in detail elsewhere. ${ }^{11}$ This is a multicenter observational cohort of current and former smokers in the US, aged between 45 and 80 years at enrollment, who reported at least 10 pack-years of cigarette smoking and self-identified as either non-Hispanic white or African-American. Local institutional ethical approval was obtained from all 21 academic clinical centers involved in participant recruitment (Appendix), and all participants provided written informed consent. For the purposes of this study, the main analyses focused on participants with COPD (defined as GOLD grade 1, 2, 3, or $4^{12}$ based on the GOLD 2011 classifications scheme), ${ }^{9}$ excluding from the main analyses controls without COPD and the group with preserved ratio and impaired spirometry. Secondary analyses for dyspnea and exacerbation risk were performed in the controls without COPD.

\section{Measurements}

Self-administered questionnaires were used to assess sociodemographic information, symptoms, and medical history at baseline as described previously. ${ }^{11}$ Sociodemographic information included age, gender, ethnicity, and education level (highest obtained). Symptoms were assessed using the modified Medical Research Council (mMRC) questionnaire on breathlessness (herein referred to as dyspnea),${ }^{13}$ and participants were stratified into two groups (mMRC $0-1$ vs $\mathrm{mMRC} \geq 2$ ) based on GOLD recommendations. ${ }^{9}$ Exacerbation risk was determined by self-reported hospitalizations occurring in the previous 12 months, and participants were grouped as low risk ( $\leq 1$ exacerbation and none requiring hospitalization) or high risk ( $\geq 2$ or $\geq 1$ requiring hospitalization). GOLD grade for airflow limitation severity was determined using spirometry (GOLD 1, mild: forced expiratory volume in 1 second $\left[\mathrm{FEV}_{1}\right] \geq 80 \%$; GOLD 2, moderate: $50 \% \leq \mathrm{FEV}_{1}<80 \%$; GOLD 3, severe: $30 \% \leq \mathrm{FEV}_{1}<50 \%$; and GOLD 4, very severe: $\mathrm{FEV}_{1}<30 \%$ ), and participants were categorized into two groups (GOLD grade 1 vs GOLD grades 2-4). Using information on dyspnea, exacerbation history, and airflow limitations, participants were classified into four GOLD groups (A, B, C, and D) according to the GOLD 2011 recommendations (A: low risk, less symptoms; B: low risk, more symptoms; C: high risk, less symptoms; D: high risk, more symptoms). ${ }^{9}$

\section{Statistical analysis \\ Age grouping analyses}

A stepwise approach was adopted to determine the age categorizations.

Step 1: A series of regression models were run to explore the relationship between age, gender, and COPD GOLD group. The interaction between age and gender was tested using a multinomial logistic regression model with gender, age (as a continuous variable), and an age-by-gender interaction term as covariates and GOLD group (B, C, and D vs A) as the outcome. A significant age-gender interaction was found (Appendix).

Step 2 (Figure 1): The best age categorizations to model the age-gender relationship were sought. Age at enrollment was initially stratified into seven groups at 5 -year increments $(\leq 50,>50-\leq 55,>55-\leq 60,>60-\leq 65,>65-\leq 70,>$ $70-75$, and $>75$ ). A univariate multinomial logistic regression model tested the relationship between gender and GOLD groups (B, C, and D vs A) within each age strata. A multivariable mixed effect regression model was also run 
A

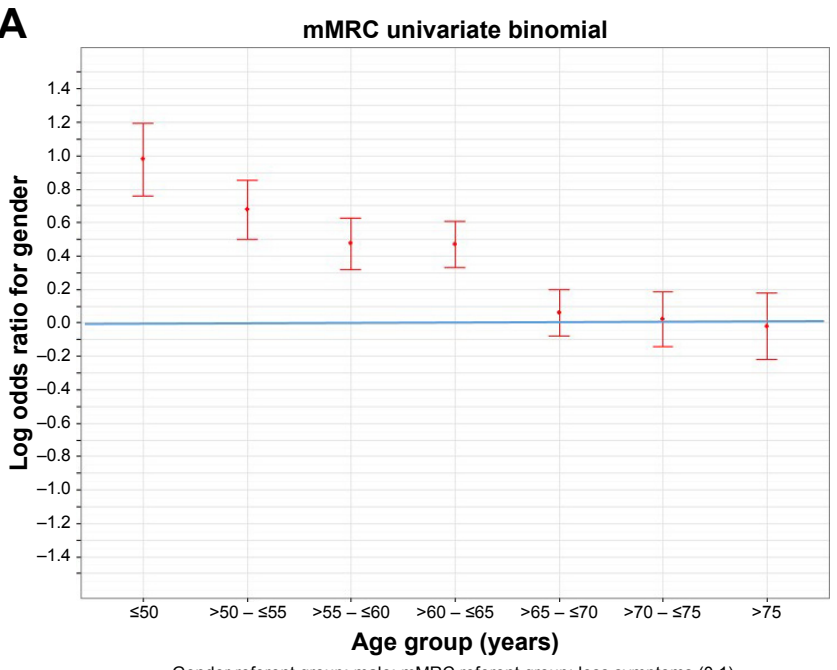

B

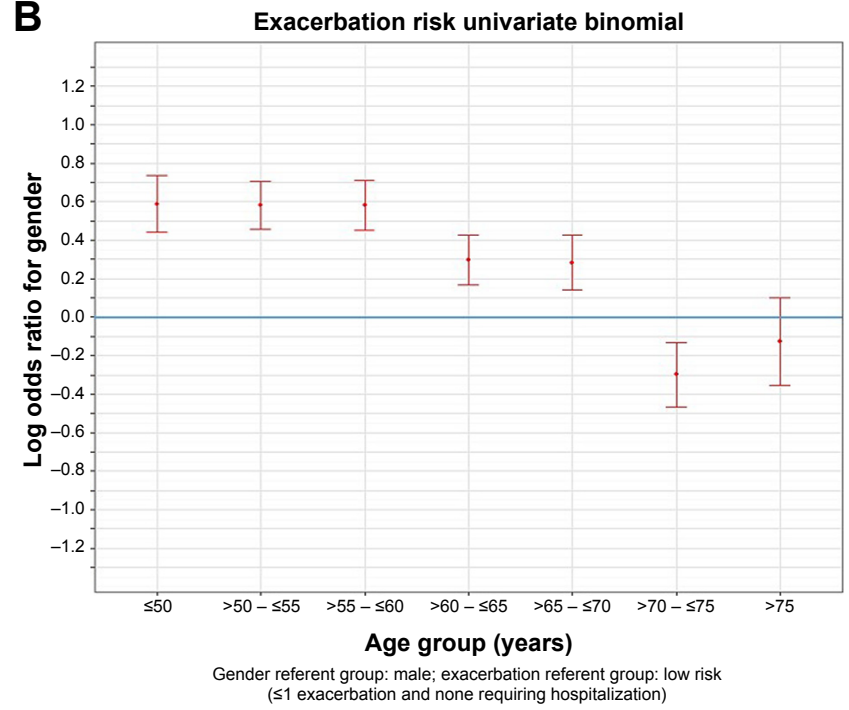

C

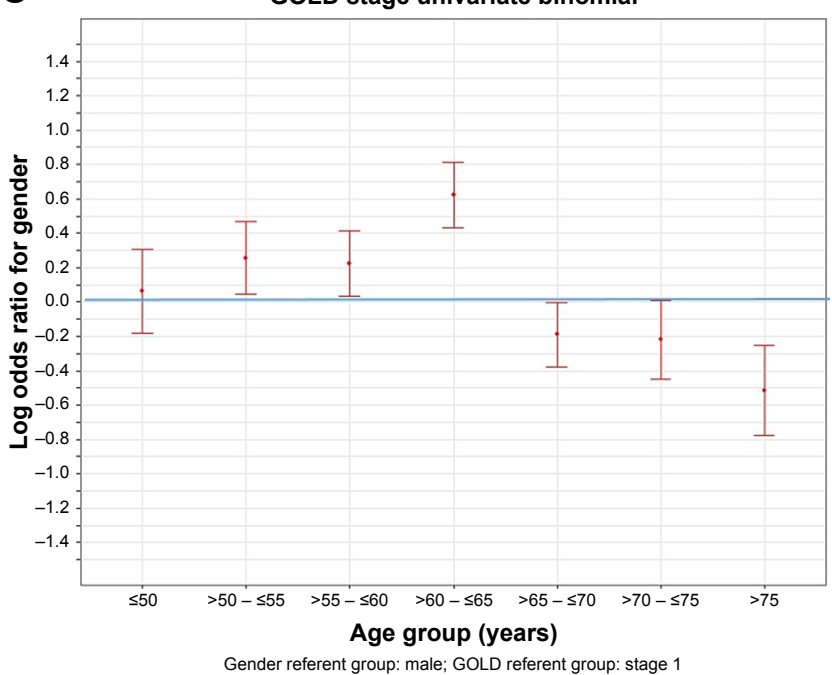

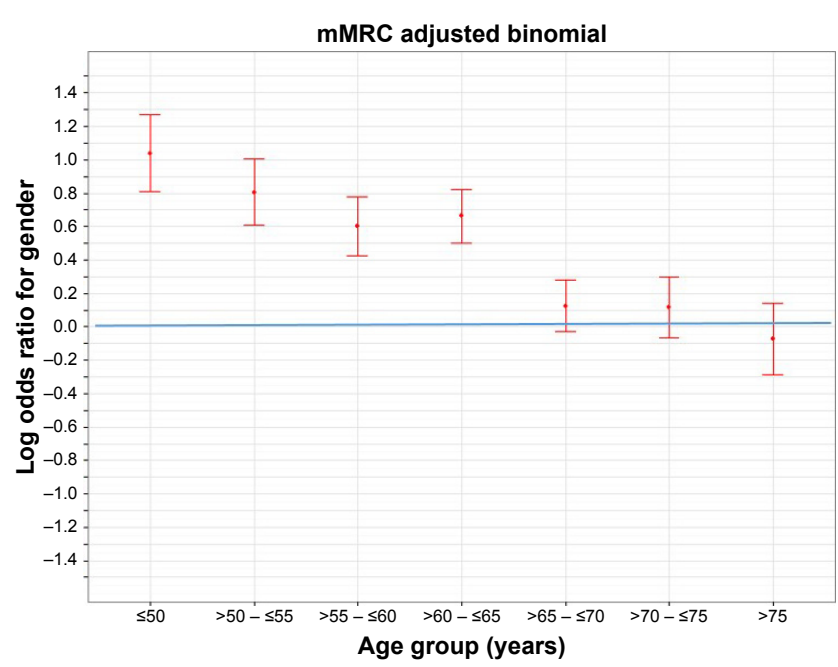
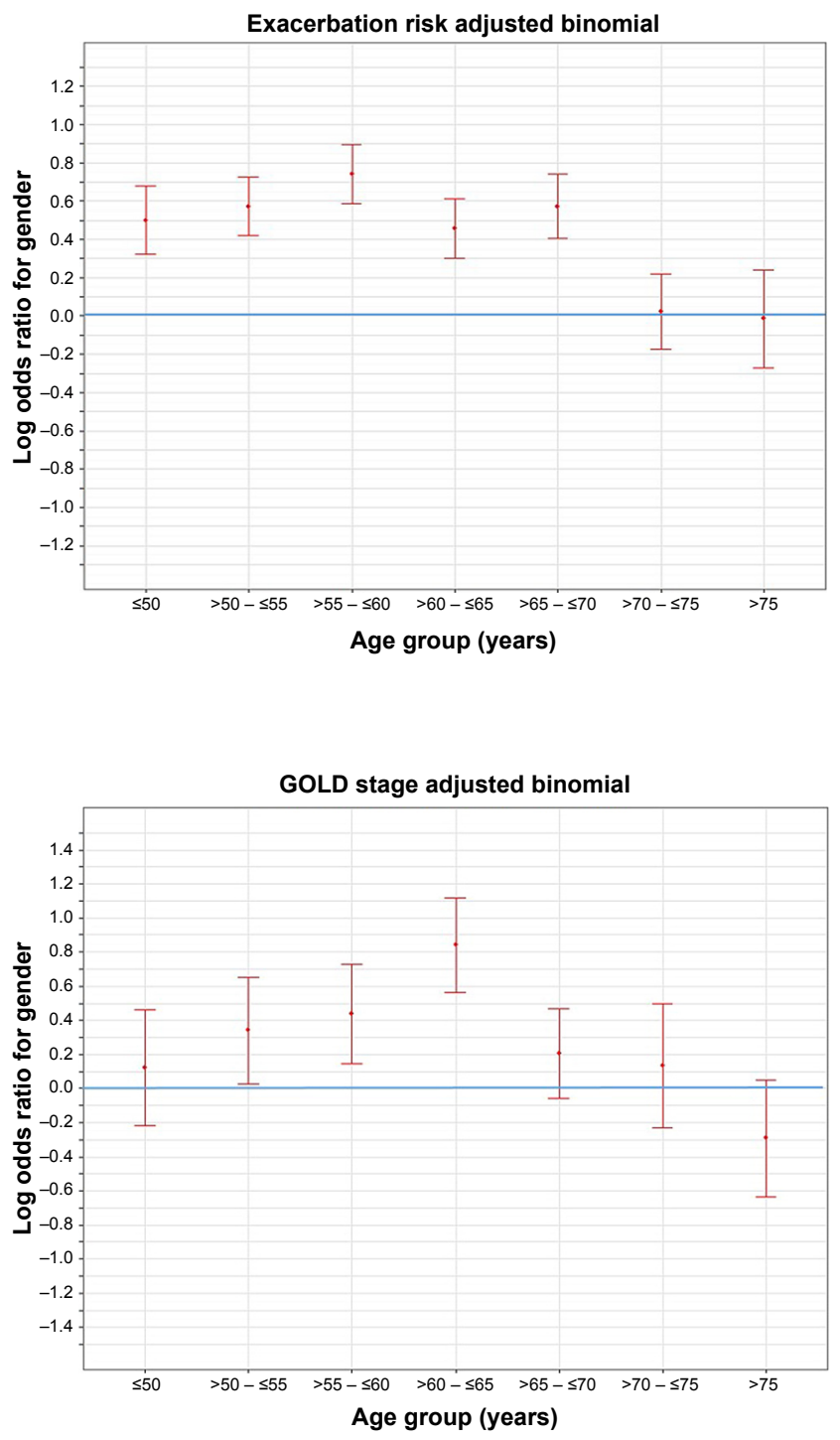

Figure I (Continued) 

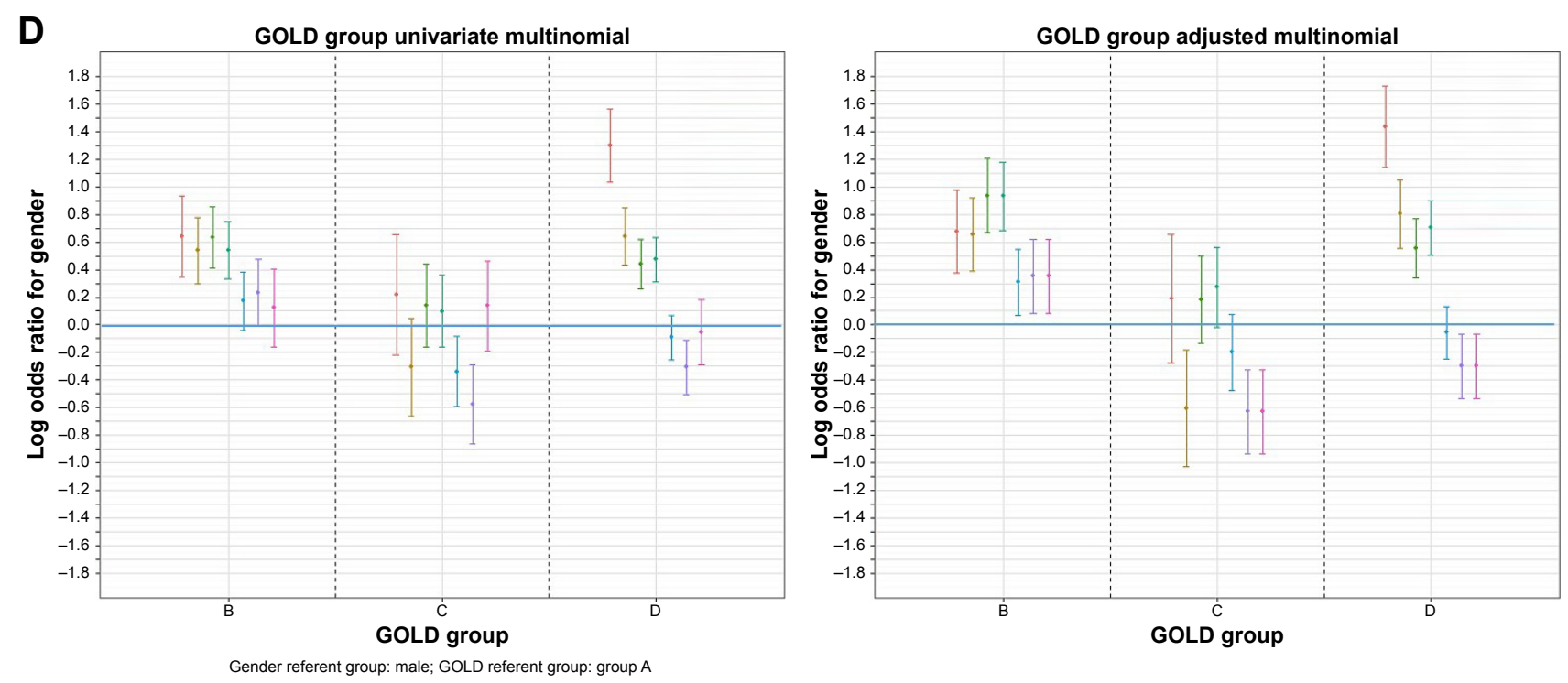

$$
\begin{aligned}
& \text { Age categories (years) } \\
& +\leq 50 \rightarrow>50-\leq 55 \rightarrow>55-\leq 60 \rightarrow>60-\leq 65+>65-\leq 70 \rightarrow>70-\leq 75+>75
\end{aligned}
$$

Figure I Association of gender with dyspnea (A), exacerbation risk (B), GOLD stage (C), and GOLD group (D) in 5-year age increments. (A) Results of the univariate and adjusted binomial logistic regression models describing the association of gender with dyspnea (measured by mMRC) in 5-year age increments. The adjusted models included age, ethnicity, level of education, pack-year smoking history, and current cigarette smoking status (as of I month prior to study entry). (B) Results of the univariate and adjusted binomial logistic regression models describing the association of gender with exacerbation risk in 5 -year age increments. The adjusted models included age, ethnicity, level of education, pack-year smoking history, and current cigarette smoking status (as of I month prior to study entry), current COPD status, FEV,\%. (C) Results of the univariate and adjusted binomial logistic regression models describing the association of gender with GOLD stage in 5-year age increments. The adjusted models included age, ethnicity, level of education, pack-year smoking history, current cigarette smoking status (as of I month prior to study entry), total emphysema (-950 Hu). (D) Results of the univariate and adjusted binomial logistic regression models describing the association of gender with GOLD group in 5-year age increments. Multinomial model adjusted for age, ethnicity, level of education, pack-year smoking history, current cigarette smoking status (as of I month prior to study entry), total emphysema (-950 Hu). Abbreviations: mMRC, modified Medical Research Council; GOLD, Global Initiative for Chronic Obstructive Lung Disease; FEV , forced expiratory volume in I second.

using the same age groupings, to ensure the results were not a reflection of confounding. Using univariate and multivariable binomial logistic regression, this method was replicated for mMRC, exacerbation risk, and GOLD stage (Figure 1A-C). All multivariable models were adjusted for age, race, education level, and smoking burden. For both the univariate and adjusted models, log odds ratios (Log-ORs) and confidence intervals (CIs) were plotted by age category (Figure 1). The use of Log-ORs in Figure 1 provides symmetric CIs, while the ORs may not.

Step 3: Plots for the association between gender and COPD GOLD group were visually inspected for each of the seven age categories, and the results, including consideration of the $95 \%$ CIs, were discussed among all authors to identify potential age groups that could be aggregated. The association between gender and GOLD group was similar for all subjects aged $<65$ and $\geq 65$ years, even after adjustment for important confounders (Figure 1D). Therefore, the age stratification $<65$ and $\geq 65$ years was used as a clinically meaningful cutoff point to evaluate the gender-specific differences.

\section{Gender differences in COPD GOLD groups and its components by age}

The demographic and clinical characteristics of participants at enrollment were described using number and percentage for categorical variables and mean and standard deviation for continuous variables. Univariate comparisons between men and women in both the younger and older groups were made using Student's $t$-test for continuous variables, Mann-Whitney $U$-test for ordinal variables, and chi-square test for categorical variables.

The primary outcome measure for this study was GOLD grade, which includes symptom burden. Mixed-effects binomial and multinomial logistic regression models were run to explore the association of gender in COPD GOLD groups ( $\mathrm{B}, \mathrm{C}$, and $\mathrm{D}$ vs $\mathrm{A}$ ) and its three components: mMRC dyspnea score, exacerbation history, and GOLD grade. Each model included categorical age (as defined from the abovementioned analyses) and an age-by-gender interaction term to assess whether the association between gender and each outcome was significantly modified by age. Results are presented using the younger age category as the reference group, and data are presented as ORs and CIs. Potential confounders 
included age, race, smoking burden, and where appropriate, education level and lung function. A random intercept at the medical center level was also included in each model to account for any differences between centers. Descriptive statistics were generated using SAS software (version 9.4) and multivariate models were performed using R Statistical Software (version 0.99.491, (C) 2009-2015 R Studio, Inc, Boston, MA, USA).

\section{Results}

\section{Patient demographic characteristics}

In total, 4,484 participants with spirometry data (GOLD grades $1-4)$ were included in the analyses. Table 1 provides a description of the study population, including key demographic and clinical characteristics parsed by age and gender. There were 2,522 individuals with COPD in the $<65$ years age group and 1,962 in the $\geq 65$ years age group. The proportion of women in each age group was similar (range: $42.8 \%-45.0 \%$ ), and the majority of participants across both men and women were from a non-Hispanic white background (range: $68.5 \%-89.4 \%$ ) and had obtained higher education (range: $56.5 \%-67.1 \%$ ). A higher proportion of men in the younger group were identified as current smokers $(p<0.001)$, although there were no gender differences in current smoking status among the older participants $(p>0.05)$.

\section{Age-grouping analysis}

A significant age-by-gender interaction was observed in the multinomial logistic regression model including age as a continuous variable with GOLD group as the outcome (Appendix). In order to further understand the relationship between age, gender, and GOLD group, univariate multinomial and adjusted multinomial logistic regression models including age as a categorical variable were performed. Graphs reporting the results of these analyses are presented in Figure 1 and support parsing the data analysis at age 65 years.

Table I Demographic and clinical characteristics of the study population by age and gender (data given as mean [SD] unless stated otherwise)

\begin{tabular}{|c|c|c|c|c|c|c|}
\hline \multirow[t]{2}{*}{ Characteristics } & \multicolumn{3}{|c|}{$<65$ years $(n=2,522)$} & \multicolumn{3}{|c|}{$\geq 65$ years $(n=I, 962)$} \\
\hline & $\begin{array}{l}\text { Males } \\
(\mathrm{n}=1,387)\end{array}$ & $\begin{array}{l}\text { Females } \\
(n=I, 135)\end{array}$ & $\begin{array}{l}p \text {-value (males } \\
\text { vs females) }\end{array}$ & $\begin{array}{l}\text { Males } \\
(n=I, I 22)\end{array}$ & $\begin{array}{l}\text { Females } \\
(n=840)\end{array}$ & $\begin{array}{l}p \text {-value (males } \\
\text { vs females) }\end{array}$ \\
\hline Age (years) & $56.8(5.3)$ & $56.8(5.5)$ & 0.917 & $71.2(4.2)$ & $71.2(4.2)$ & 0.791 \\
\hline Race & & & $0.40 \mathrm{I}$ & & & 0.014 \\
\hline Non-Hispanic white, n (\%) & $950(68.5)$ & $795(70.0)$ & & $\mathrm{I}, 003(89.4)$ & $720(85.7)$ & \\
\hline Black/African-American, n (\%) & $437(31.5)$ & $340(30.0)$ & & $119(10.6)$ & $120.0(\mid 4.3)$ & \\
\hline Education level & & & 0.741 & & & 0.001 \\
\hline High school or less, n (\%) & $603(43.5)$ & $486(42.8)$ & & $368(32.8)$ & $334(39.8)$ & \\
\hline Higher education, n (\%) & $784(56.5)$ & $649(57.2)$ & & $753(67.1)$ & $506(60.2)$ & \\
\hline Current cigarette smoker & & & $<0.00$ I & & & 0.902 \\
\hline Yes, n (\%) & $88 I(63.5)$ & $647(57.0)$ & & $239(21.3)$ & I $77(2||)$. & \\
\hline No, n (\%) & $506(36.5)$ & $488(43.0)$ & & $883(78.7)$ & $663(78.9)$ & \\
\hline $\begin{array}{l}\text { Cigarettes smoked among } \\
\text { current smokers (per day) }\end{array}$ & $17.7(10.6)$ & $16.4(10.5)$ & 0.003 & $17.5(12.1)$ & $12.8(9.5)$ & $<0.001$ \\
\hline $\mathrm{FEV}, \%$ predicted & $60.5(23.2)$ & $57.75(22.4)$ & 0.002 & $53.1(22.5)$ & $57.6(22.3)$ & $<0.001$ \\
\hline Dyspnea & & & $<0.00 \mathrm{I}$ & & & 0.693 \\
\hline Less severe, n (\%) & $654(47.2)$ & $378(33.3)$ & & $47 \mid(42.0)$ & $346(4 \mid .2)$ & \\
\hline More severe, n (\%) & $728(52.5)$ & $754(66.4)$ & & $649(57.8)$ & 491 (58.5) & \\
\hline Exacerbation risk & & & $<0.00 \mathrm{I}$ & & & 0.849 \\
\hline Lower, n (\%) & I,038 (74.8) & $750(66.1)$ & & $859(76.6)$ & $640(76.2)$ & \\
\hline Higher, n (\%) & $349(25.2)$ & 385 (33.9) & & $263(23.4)$ & $200(23.8)$ & \\
\hline GOLD Grade & & & 0.012 & & & $<0.001$ \\
\hline Grade I, n (\%) & 303 (21.9) & $192(16.9)$ & & $154(13.7)$ & $145(17.3)$ & \\
\hline Grade $2-4, \mathrm{n}(\%)$ & I,084 (78.I) & $943(83.1)$ & & $968(86.3)$ & $695(82.7)$ & \\
\hline GOLD group & & & $<0.00 \mathrm{I}$ & & & 0.054 \\
\hline Group A & $530(38.2)$ & $303(26.7)$ & & $335(29.9)$ & $266(31.7)$ & \\
\hline Group B & $230(16.6)$ & $232(20.4)$ & & $164(14.6)$ & $162(19.3)$ & \\
\hline Group C & $129(9.3)$ & $78(6.9)$ & & $138(12.3)$ & $83(9.9)$ & \\
\hline Group D & 498 (35.9) & $522(46.0)$ & & $485(43.2)$ & $329(39.2)$ & \\
\hline
\end{tabular}

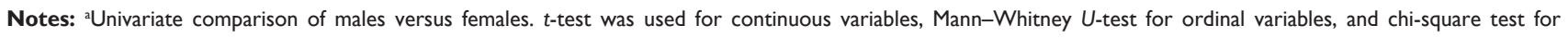
categorical variables.

Abbreviations: $\mathrm{FEV}_{1}$, forced expiratory volume in I second; GOLD, Global Initiative for Chronic Obstructive Lung Disease. 


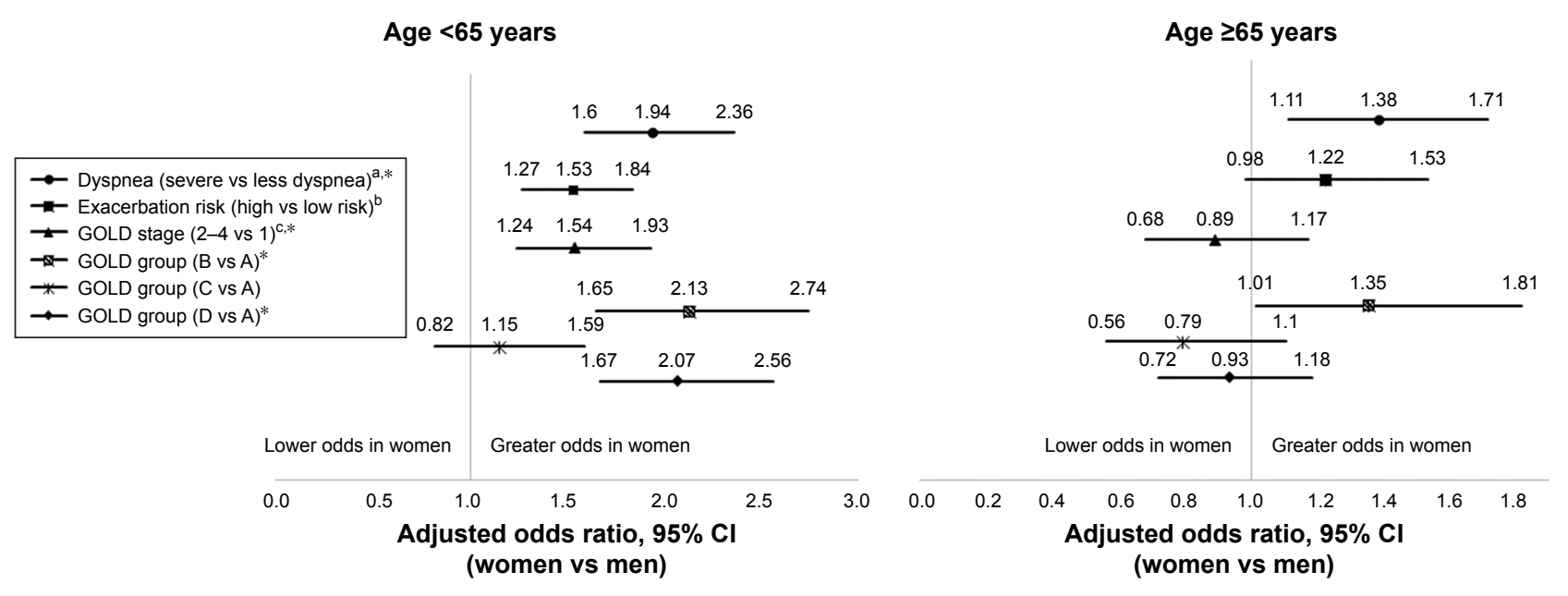

Figure 2 Adjusted ORs and $95 \% \mathrm{Cls}$ for the association of female gender with dyspnea, exacerbation risk, GOLD grade, and GOLD group in all participants aged $<65$ years and $\geq 65$ years. ${ }^{2}$ Adjusted for age, age $\times$ gender, race, current cigarette smoking status (as of I month ago), FEV, $\%$ predicted. Age $<65$ years: $n=25$ I 5 ; Age $\geq 65$ years: $n=1956$. ${ }^{b}$ Adjusted for age, age $\times$ gender, level of education, race, current cigarette smoking status (as of I month ago), number of cigarettes smoked per day, FEV $\%$ predicted. Age $<65$ years: $n=2522$; Age $>65$ years: $n=1961$. 'Adjusted for age, age $\times$ gender, level of education, race, current cigarette smoking status (as of I month ago), number of cigarettes smoked per day. Age $<65$ years: $n=2522$; Age $>65$ years: $n=1961$. *Age-by-Gender interaction is significant, $p<0.05$.

Abbreviations: OR, odds ratio; GOLD, Global Initiative for Chronic Obstructive Lung Disease.

\section{Multivariate models for gender differences in COPD Dyspnea}

Younger women with COPD had 1.94(95\% CI 1.60-2.36) higher odds of reporting severe dyspnea compared to younger men with COPD. A significant age-by-gender interaction was also observed ( $p=0.02$ ). Specifically, the association between gender and symptom reporting was attenuated among older participants. Older women with COPD remained significantly more likely to report severe dyspnea compared to older men with COPD (OR 1.38, 95\% CI 1.11-1.71). These results are plotted in Figures 1 and 2, and the full models are provided in Appendix. Among 4,387 individuals without COPD (2,319 men, 2,068 women), both younger and older women reported more severe dyspnea at baseline (unadjusted comparison $p<0.01$, data not shown).

\section{Exacerbation risk}

Younger women with COPD had 1.53 (95\% CI 1.27-1.84) higher odds for exacerbation risk compared to younger men with COPD. The association was weaker (OR 1.22, 95\% CI $0.98-1.53$ ) and non-significant among older participants, and no age-by-gender interaction was observed $(p=0.13)$. These results are plotted in Figures 1 and 2, and the full models are provided in Appendix. Among 4,383 individuals without COPD (2,320 men, 2,063 women), both older and younger women reported more respiratory exacerbations at baseline (unadjusted comparison $p<0.005$, data not shown).

\section{GOLD grade for airflow limitation severity}

Younger women had 1.54 (95\% CI 1.24-1.93) higher odds of having more severe airflow limitations (GOLD grade 2-4) compared to younger men. A significant age-bygender interaction was also observed $(p=0.002)$. Specifically, the association between gender and airflow limitation was attenuated among the older participant group. In this group, women had lower odds (OR $0.89,95 \%$ CI $0.68-1.17$ ) of severe airflow limitations compared to men, although this association was not significant. These results are plotted in Figures 1 and 2, and the full models are provided in Appendix.

\section{GOLD group}

Younger women had higher odds of being classified in GOLD group D versus A compared to younger men (OR 2.07, 95\% CI 1.67-2.56), despite lower pack-years of smoking. This relationship was attenuated by age; among the older age group, women had lower odds of being classified in GOLD group D versus A (OR 0.93, 95\% CI 0.72-1.18), although this association was not significant. Younger women also had higher odds of being classified in GOLD group B versus A (OR 2.13, 95\% CI 1.65-2.74) compared to men. This relationship was significantly modified by age $(p=0.021)$ such that the relationship was less strong in the older group. However, older women remained significantly more likely to be classified in GOLD group B versus A compared to older men (OR 1.35, 95\% CI 1.01-1.81). These results are plotted in Figures 1 and 2, and the full models are provided in Appendix.

\section{Discussion}

This analysis of data from $>4,400$ individuals with COPD demonstrated that younger women were more likely to manifest severe dyspnea, more severe airflow limitation, greater risk for 
exacerbations, and subsequently more severe GOLD classification than younger men. These associations with gender were less pronounced among older adults ( $\geq 65$ years), particularly with respect to dyspnea, airflow limitations, and GOLD classification. Despite this, older women with COPD were more likely to experience dyspnea and present with more severe COPD (GOLD B vs A) than older men. The results suggest that women are more significantly burdened by the symptoms of COPD than men and that younger women may be particularly susceptible.

These findings are consistent with previous work showing that the female gender is associated with more severe manifestations of COPD. In a sample of $>6,000$ participants in the TORCH study, women with COPD reported worse MRC dyspnea scores at baseline, and over a 3-year follow-up period had a $25 \%$ higher rate of exacerbations and a shorter time to first exacerbation compared to men. ${ }^{5}$ In a separate population-based study, women identified as having COPD reported significantly more dyspnea than male participants, and a significantly greater proportion of women reported ever having an exacerbation compared to men. ${ }^{14}$ The results of this study extend this previous work to show gender differences vary across the life course and persist in clinically meaningful combined COPD profiles (GOLD groups). Specifically, women in this study were more likely than similarly aged men to be classified in GOLD group B compared to group A, a group identified as being at a lower risk for exacerbations and airflow limitations, but experiencing more severe symptoms. Equally, among the younger age group ( $<65$ years), women were more than twice as likely to be categorized into GOLD group D compared to group A. GOLD group D classified individuals are identified both as being at high risk for exacerbations and airflow limitations and having more severe symptoms. This is clinically relevant given that these groups have been associated with COPDrelated hospitalizations and mortality. ${ }^{15}$

The results presented here also build on previous work to show gender differences in COPD are modified by age and appear most pronounced among younger adults ( $<65$ years), particularly with respect to dyspnea, exacerbation risk, and COPD combined profiles (GOLD B group vs group A and GOLD group D vs group A). In this study, a detailed approach was adopted to identify the age categorizations for use in the final models. Age categorization analyses supported delineation of age 65 years as a clinically meaningful cut-point from which to identify gender-related differences in the clinical manifestations of COPD. This information could inform gender-specific clinical management of COPD, while providing a meaningful demarcation from which future work can continue to understand gender-related differences in lung disease through adulthood.
Reasons for the observed gender and age differences are not yet fully understood and may involve multiple interacting mechanisms operating at a physiological, behavioral, and/ or genetic level. Gender differences in the report of dyspnea have been suggested to have a variety of explanations, some of which have included differences in rates of anxiety and depression. ${ }^{16}$ A recent report from the Swedish CardioPulmonarybioImage Study (SCAPIS) has suggested and supported a mechanistic reason for the increased reports of breathlessness in a general population study of men and women is due to lower absolute lung volumes in women and found no association of breathlessness with anxiety, depression, and menopause. ${ }^{17}$ Cigarette smoke is the most important known risk factor for COPD, ${ }^{18}$ and one possibility is that women may be more susceptible to its toxicity, ${ }^{19}$ with increased symptoms and lung function decline. There is evidence that estrogen, a female sex hormone, may mediate sex differences in smoke metabolism, causing women to be more physiologically susceptible. ${ }^{20}$ Importantly, the peri-menopausal time has been associated with accelerated lung function decline, further supporting a pivotal role for differential impact of cigarette smoking as a contributor to some of the age by gender findings we observe. ${ }^{21}$ Of particular importance may be that women tend to have anatomically smaller airways than men, such that for each cigarette smoked there is a proportionately greater exposure to toxins. ${ }^{22} \mathrm{~A}$ sex-specific genetic/epigenetic contribution can also not be ruled out, especially complex three-way interactions between smoking, gender, and age. Indeed, the majority of cases of severe early-onset COPD are known to be women, and among relatives of these individuals who smoke, women are more likely than men to present with severe airway limitations. ${ }^{7}$ A recent sex-stratified genome-wide association study revealed sex-specific association of the CELSR1 gene with COPD in women and not men. This gene is relevant to development, suggesting that lung development differences may contribute to later life gender differences in COPD. ${ }^{23}$ In addition, the human lung transcriptome has been demonstrated to have both age and gender associations, suggesting that the genes contributing to lung health and disease during adulthood will vary across the life course. ${ }^{24}$ Gender differences in the reporting, access, or response to available treatment modalities is also a possibility, ${ }^{25,26}$ although more work is needed to better understand the underlying pathways.

Regardless of the mechanisms driving the age-related gender differences in the presentation of COPD, the results of this study have clear implications. From a scientific perspective, more research needs to be done to understand the biology of sex and gender differences in pulmonary disease. Clinically, physicians need to be made aware of the 
significant female burden of COPD, with a specific emphasis on treatable symptoms, especially for women in the fifth and sixth decades of life, during which the menopausal transition may increase respiratory decline. ${ }^{26}$ Increased awareness could lead to earlier diagnosis and treatment and is particularly important to consider in light of evidence that a gender bias exists in the diagnosis of COPD, with women less likely to be diagnosed and more likely to report diagnostic delay. ${ }^{27}$ Women with COPD may benefit from more intensive and earlier management of chronic bronchitis related to cough and phlegm. In the Rotterdam Study, women with chronic bronchitis had significantly worse survival. ${ }^{28}$ Additionally, a recent analysis of 2,749 subjects from the Coronary Artery Risk Development in Young Adults (Cardia) Lung Study demonstrated that persistent respiratory symptoms in young adults were associated with increased lung function decline, incident obstructive spirometry findings, and increased odds of emphysema. ${ }^{29}$ The present findings indicate that factors influencing the evaluation and treatment of individuals with COPD should include gender in addition to the more commonly recognized smoking status, age, and ethnicity. Indeed, early and targeted interventions aimed at symptom alleviation in younger women may improve health outcomes and functioning in this group.

This study has several limitations. First, information on medical history was self-reported, so may have been subject to recall bias. Furthermore, participants were volunteers recruited from academic clinical centers so may not be representative of patients with COPD in the general population. Despite this, it is important to consider that this was a large, extensively phenotyped sample of current and former smokers. However, this study is unable to address the issue of combined age and sex features for COPD in life-time never smokers. ${ }^{30}$ While this study benefited from including both non-Hispanic white and African-American participants, we are underpowered to determine whether the observed gender by age effects are also modified by race. This is important given that African-Americans are known to be at higher risk of severe early-onset COPD, ${ }^{7}$ and so the role of a three-way interaction between age, race, and gender is an important consideration for future research.

\section{Conclusion}

This study, in a large contemporary cohort of current and former smokers with COPD, suggests that women are more likely to be significantly burdened by the symptoms of COPD than men, and this effect appears most pronounced among younger women. Although prior results have highlighted important sex and gender differences in men and women with COPD, the modeling of age by gender interactions across the fifth through eighth decades of life is a point of novelty and highlights the clinical imperative to more effectively and thoroughly recognize and manage respiratory symptoms in women. More research is needed to fully understand the pathogenesis of increased severity of COPD in women and to develop gender-targeted management approaches ${ }^{24,31}$ to improve outcomes across the life course for both men and women with COPD.

\section{Acknowledgments}

The authors would like to gratefully acknowledge the contribution of all COPDGene participants and field investigators for their willingness to contribute to medical research. We also acknowledge the contribution of Drs Robert Fogel and Amy Blickensderfer from Novartis, and Jack Ishak, Selin Cooper, and Laura McDonald from Evidera, who all provided expert opinion during the data analysis and interpretation of the results, and Laura McDonald for drafting the manuscript. COPDGene study was funded by National Institutes of Health (NIH) grants U01HL089856 and U01HL089897.The COPDGene Study (NCT00608764) is also supported by the COPD Foundation through contributions made to an Industry Advisory Board comprised of AstraZeneca, Boehringer Ingelheim, GlaxoSmithKline, Novartis, Pfizer, Siemens and Sunovion. Neither the NIH nor the Industry Advisory Board had a role in the study design, data collection, data analysis, interpretation of the data, writing of the report or the decision to submit the paper for publication. The content is solely the responsibility of the authors and does not necessarily represent the official views of the National Heart, Lung, And Blood Institute or the National Institutes of Health.

\section{Disclosure}

DL DeMeo, MD, reports receiving grants from the National Institutes of Health and consultation fees from Novartis. $\mathrm{S}$ Ramagopalan, $\mathrm{PhD}$, was an employee of Evidera Inc. (scientific consulting) at the time of the study. A Kavati, PhD, is an employee of Novartis Pharmaceuticals. A Vegesna, PharmD, is an employee of Novartis Pharmaceuticals. MK Han, MD, reports consulting income from GSK, Boehringer Ingelheim, AstraZeneca, Sunovion she also reports research support from GSK and Novartis. A Yadao, MD, is an employee of Novartis Pharmaceuticals. TK Wilcox, $\mathrm{PhD}$, is an employee of Evidera Inc. (scientific consulting). BJ Make, MD, related to the topic of COPD, reports funding from the National Heart, Lung, and Blood Institute for the COPDGene study, grants, and 
personal fees from Boehringer Ingelheim, AstraZeneca; personal fees and other from Spiration, Consensus Medical Education, Integrity Medical Education, Mt Sinai Medical Center, WebMD, Up-To-Date, National Jewish Health; grants, personal fees, and other from GlaxoSmithKline, Sunovian; personal fees from Novartis, CSL Bering, Verona; other from SPIRE Learning, American College of Chest Physicians, Projects in Knowledge, Hybrid Communications, Peer Review Institute, National Jewish Health, Cleveland Clinic; grants from Pearl; grants and other from Medscape, outside the submitted work. The authors report no other conflicts of interest in this work.

\section{References}

1. Centers for Disease Control and Prevention. Deaths, percent of total deaths, and death rates for the 15 leading causes of death in 5-year age groups, by race and sex: United States; 2013. Available from: http://www. cdc.gov/nchs/data/dvs/LCWK1_2013.pdf. Accessed March 23, 2016.

2. Mannino DM, Homa DM, Akinbami LJ, Ford ES, Redd SC. Chronic obstructive pulmonary disease surveillance - United States, 1971-2000. MMWR Surveill Summ. 2002;51(6):1-16.

3. Wise RA. Changing smoking patterns and mortality from chronic obstructive pulmonary disease. Prev Med. 1997;26(4):418-421.

4. Sorheim IC, Johannessen A, Gulsvik A, Bakke PS, Silverman EK, DeMeo DL. Gender differences in COPD: are women more susceptible to smoking effects than men? Thorax. 2010;65(6):480-485.

5. Celli B, Vestbo J, Jenkins CR, et al. Sex differences in mortality and clinical expressions of patients with chronic obstructive pulmonary disease. The TORCH experience. Am J Respir Crit Care Med. 2011; 183(3):317-322.

6. Christensen VL, Rustoen T, Cooper BA, et al. Distinct symptom experiences in subgroups of patients with COPD. Int J Chron Obstruct Pulmon Dis. 2016;11:1801-1809.

7. Silverman EK, Weiss ST, Drazen JM, et al. Gender-related differences in severe, early-onset chronic obstructive pulmonary disease. Am J Respir Crit Care Med. 2000;162(6):2152-2158.

8. Foreman MG, Zhang L, Murphy J, et al. Early-onset chronic obstructive pulmonary disease is associated with female sex, maternal factors, and African American race in the COPDGene Study. Am J Respir Crit Care Med. 2011;184(4):414-420.

9. Vestbo J, Hurd SS, Agusti AG, et al. Global strategy for the diagnosis, management, and prevention of chronic obstructive pulmonary disease: GOLD executive summary. Am J Respir Crit Care Med. 2013;187(4):347-365.

10. Han MK, Postma D, Mannino DM, et al. Gender and chronic obstructive pulmonary disease: why it matters. Am J Respir Crit Care Med. 2007;176(12):1179-1184.

11. Regan EA, Hokanson JE, Murphy JR, et al. Genetic epidemiology of COPD (COPDGene) study design. COPD. 2010;7(1):32-43.

12. Rabe KF, Hurd S, Anzueto A, et al. Global strategy for the diagnosis, management, and prevention of chronic obstructive pulmonary disease: GOLD executive summary. Am J Respir Crit Care Med. 2007;176(6): $532-555$.
13. Brooks S. Surveillance for respiratory hazards. ATS News. 1982;8: 12-16.

14. Lopez Varela MV, Montes de Oca M, Halbert RJ, et al. Sex-related differences in COPD in five Latin American cities: the PLATINO study. Eur Respir J. 2010;36(5):1034-1041.

15. Agusti A, Edwards LD, Celli B, et al. Characteristics, stability and outcomes of the 2011 GOLD COPD groups in the ECLIPSE cohort. Eur Respir J. 2013;42(3):636-646.

16. Di Marco F, Verga M, Reggente M, et al. Anxiety and depression in COPD patients: the roles of gender and disease severity. Respir Med. 2006;100(10):1767-1774. Epub 2006 Mar 13.

17. Ekström M, Sundh J, Schiöler L, et al. Absolute lung size and the sex difference in breathlessness in the general population. PLoS One. 2018;13(1):e0190876.

18. Mannino DM, Buist AS. Global burden of COPD: risk factors, prevalence, and future trends. Lancet. 2007;370(9589):765-773.

19. Sin DD, Cohen SB, Day A, Coxson H, Pare PD. Understanding the biological differences in susceptibility to chronic obstructive pulmonary disease between men and women. Proc Am Thorac Soc. 2007;4(8):671-674.

20. Ben-Zaken Cohen S, Pare PD, Man SF, Sin DD. The growing burden of chronic obstructive pulmonary disease and lung cancer in women: examining sex differences in cigarette smoke metabolism. Am J Respir Crit Care Med. 2007;176(2):113-120.

21. Triebner K, Matulonga B, Johannessen A, et al. Menopause is associated with accelerated lung function decline. Am J Respir Crit Care Med. 2017; 195(8):1058-1065.

22. Becklake MR, Kauffmann F. Gender differences in airway behaviour over the human life span. Thorax. 1999;54(12):1119-1138.

23. Hardin M, Cho MH, Sharma S, et al. Sex-based genetic association study identifies celsr1 as a possible chronic obstructive pulmonary disease risk locus among women. Am J Respir Cell Mol Biol. 2017;56(3): $332-341$

24. Li X, Obeidat M, Zhou G, et al. Responsiveness to ipratropium bromide in male and female patients with mild to moderate chronic obstructive pulmonary disease. EBioMedicine. 2017;19:139-145.

25. Scanlon PD, Connett JE, Waller LA, et al. Smoking cessation and lung function in mild-to-moderate chronic obstructive pulmonary disease. The Lung Health Study. Am J Respir Crit Care Med. 2000;161(2 Pt 1): 381-390.

26. Martinez CH, Raparla S, Plauschinat CA, et al. Gender differences in symptoms and care delivery for chronic obstructive pulmonary disease. $J$ Womens Health (Larchmt). 2012;21(12):1267-1274.

27. Chapman KR, Tashkin DP, Pye DJ. Gender bias in the diagnosis of COPD. Chest. 2001;119(6):1691-1695.

28. Lahousse L, Seys LJM, Joos GF, Franco OH, Stricker BH, Brusselle GG. Epidemiology and impact of chronic bronchitis in chronic obstructive pulmonary disease. Eur Respir J. 2017;50(2):1602470.

29. Kalhan R, Dransfield MT, Colangelo LA, et al. Respiratory symptoms in young adults and future lung disease: the CARDIA Lung Study. Am J Respir Crit Care Med. Epub 2018 Jan 25.

30. Tan WC, Sin DD, Bourbeau J, et al. Characteristics of COPD in neversmokers and ever-smokers in the general population: results from the CanCOLD study. Thorax. 2015;70(9):822-829.

31. Tsiligianni I, Mezzi K, Fucile S, et al. Response to indacaterol/ glycopyrronium (IND/GLY) by sex in patients with COPD: a pooled analysis from the IGNITE program. COPD. 2017;14(4):375-381.

International Journal of COPD

\section{Publish your work in this journal}

The International Journal of COPD is an international, peer-reviewed journal of therapeutics and pharmacology focusing on concise rapid reporting of clinical studies and reviews in COPD. Special focus is given to the pathophysiological processes underlying the disease, intervention programs, patient focused education, and self management protocols.

This journal is indexed on PubMed Central, MedLine and CAS. The manuscript management system is completely online and includes a very quick and fair peer-review system, which is all easy to use. Visit http://www.dovepress.com/testimonials.php to read real quotes from published authors. 